\title{
Horizon Length and Fuel Equivalents for Fuel-optimal Look-ahead Control
}

\author{
Erik Hellström* Jan Åslund* Lars Nielsen * \\ * Linköping University, Linköping Sweden
}

\begin{abstract}
:
Recent studies from several authors show that it is possible to lower the fuel consumption for heavy trucks by utilizing information about the road topography ahead of the vehicle. The approach in these studies is receding horizon control where horizon length and residual cost are main topics. To approach these topics, fuel equivalents previously introduced based on physical intuition are given a mathematical interpretation in terms of Lagrange multipliers. Measures for the suboptimality, caused by the truncated horizon and the residual cost approximation, are defined and evaluated for different routes and parameters.
\end{abstract}

Keywords: automotive control, intelligent cruise control, multipliers, predictive control

\section{INTRODUCTION}

The scenario of look-ahead control is a heavy truck operating on varying terrain, and there is road data onboard so that the road slope ahead of the vehicle is known. The objective used is to minimize the fuel mass $M$ required for a drive mission with a given maximum trip time $T_{0}$ :

$$
\begin{gathered}
\text { minimize } M \\
\text { subject to } T \leq T_{0}
\end{gathered}
$$

A receding horizon control ( $\mathrm{RHC}$ ) approach has successfully been used for the solution, and experimental evidence confirms that it is possible to improve the fuel economy of heavy trucks by this approach (Hellström et al., 2009). RHC is a general method to find an approximation for the optimal control law by solving on-line, at each time step, a finite horizon optimal control problem (see, e.g., the survey paper Mayne et al., 2000). In this method, it is crucial how to select the residual cost at the end of the finite horizon and how to select a proper horizon length to balance between computational complexity and suboptimality. These two topics are in this paper given a thorough investigation that is independent of the method of solving the optimal control problem in each time step.

The line of investigation is to consider (P1) but also the reformulation

$$
\text { minimize } M+\beta T
$$

where $\beta$ determines the compromise between fuel mass and trip time. Problem (P1) is solved in Hellström et al. (2010a) by developing an efficient algorithm for (P2). The RHC approach is taken in recent papers on the problem (P1) for heavy trucks (Terwen et al., 2004; Hellström et al., 2007, 2009; Huang et al., 2008; Passenberg et al., 2009). These works utilize the reformulation (P2) except for Terwen et al. (2004) where cruise control rather than fuel-optimal control is considered by adding a quadratic penalty on deviations from a cruise speed in (P1). Although the method of solution in each time step differs in these studies, the length of the horizon and the residual cost are important common issues. A residual cost is selected and explained by Hellström et al. (2010a) that is linear in kinetic energy where the gradient $\gamma$ represents the trade-off between fuel mass and kinetic energy at the end of the horizon. The deviation from optimality thus depends on the length of the horizon and the choice of the fuel equivalence factors $(\beta, \gamma)$, and the issue of suboptimality is studied here.

The paper starts out by formulating models of the longitudinal vehicle dynamics that follows the treatment in previous papers (Hellström et al., 2009, 2010a). Next lookahead control is recalled, and measures are introduced for the suboptimality due to the truncated horizon. The following two sections treat the fuel equivalents. These were based on physical intuition in the prior papers but a clear mathematical interpretation is added here by relating to well established optimal control theory. A quantitative study is then performed to evaluate how the degree of suboptimality depends on the horizon length, vehicle mass, and road characteristics.

\section{MODEL}

The dynamics of the vehicle is modeled by considering the motion in one dimension, see Fig. 1. The propelling force is denoted by $F_{p}$. The drag force is denoted by $F_{d}(s, v)$ and is dependent on position $s$ and velocity $v$. The velocity dynamics is given by Newton's second law of motion,

$$
m v \frac{d v}{d s}=F_{p}-F_{d}(s, v)
$$

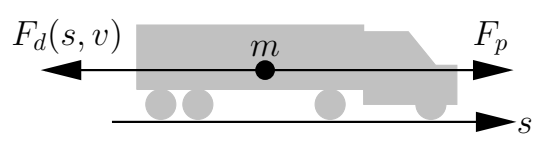

Fig. 1. A vehicle moving in one dimension.

In the following, the full model and the basic model are presented. The full model has two states and three control signals whereas the basic model is an approximation of the full model and has one state and one control. 


\subsection{Full model}

A model of the longitudinal dynamics is formulated for a stiff driveline (Kiencke and Nielsen, 2005). Gear shifting is considered and the ratio $i$ and efficiency $\eta$ are functions of the current gear. The engine torque $T_{e}$ is given by a look-up table from measurements. The states are velocity $v$ and engaged gear $g$, and the controls are fueling $u_{\mathrm{f}}$, braking $u_{\mathrm{b}}$ and gear $u_{\mathrm{g}}$. The road slope is given by $\alpha(s)$ and the brake torque is denoted by $T_{b}$. The forces in (1) are then

$$
\begin{gathered}
F_{p}=\frac{1}{c_{m} r_{w}}\left(i \eta T_{e}\left(v, g, u_{\mathrm{f}}\right)-T_{b}\left(u_{\mathrm{b}}\right)\right) \\
F_{d}(s, v)=\frac{1}{c_{m}}\left(F_{a}(v)+F_{r}(s)+F_{g}(s)\right)
\end{gathered}
$$

where $c_{m}=1+\frac{I_{l}+\eta i^{2} I_{e}}{m r_{w}^{2}}$ is a mass factor. The models of the resisting forces and all model parameters are explained in Table 1 and 2 respectively.

Table 1. Longitudinal forces.

\begin{tabular}{lll} 
Force & Explanation & Expression \\
\hline$F_{a}(v)$ & Air drag & $\frac{1}{2} c_{w} A_{a} \rho_{a} v^{2}$ \\
$F_{r}(s)$ & Rolling resistance & $m g_{0} c_{r} \cos \alpha(s)$ \\
$F_{g}(s)$ & Gravitational force & $m g_{0} \sin \alpha(s)$ \\
\hline
\end{tabular}

Table 2. Truck model parameters.

\begin{tabular}{clcl}
\hline$I_{l}$ & Lumped inertia & $c_{w}$ & Air drag coefficient \\
$I_{e}$ & Engine inertia & $A_{a}$ & Cross section area \\
$m$ & Vehicle mass & $\rho_{a}$ & Air density \\
$r_{w}$ & Wheel radius & $c_{r}$ & Rolling res. coeff. \\
$g_{0}$ & Gravity constant & & \\
\hline
\end{tabular}

The fuel consumption is given by integrating the mass flow of fuel $M^{\prime}(\mathrm{g} / \mathrm{m})$,

$$
M^{\prime}=\frac{n_{c y l}}{2 \pi n_{r}} \frac{i}{r_{w}} u_{\mathrm{f}}
$$

where $n_{c y l}$ is the number of cylinders, $n_{r}$ is the number of engine revolutions per cycle, and $u_{\mathrm{f}}$ is grams of fuel per engine cycle and cylinder.

\subsection{Basic model}

A basic model is derived as an approximation of the full model for the purpose of analytical calculations later on. A constant gear and no braking is assumed, the state is velocity $v$ and the control is fueling $u$.

Measurements of engine torque $T_{e}$ as a function of fueling $u$ for a diesel engine typically show an approximate affine relation, see, e.g., Hellström et al. (2010a) for an experimental validation. Such an approximation can be interpreted as a Willans description (Guzzella and Sciarretta, 2005). Denote an estimated gradient of this relation by $c_{e}$, then

$$
\Delta u=c_{e} \Delta T_{e}
$$

holds approximately. For the basic model, it is assumed that the fueling is proportional to the torque with $c_{e}$ being the constant of proportionality (the drag torque offset $d_{e}$ is included in the drag forces without changing the model structure). The dependence on the road slope $\alpha$ is approximated to first order ( $\alpha$ is typically a few percent). The forces in (1) become

$$
\begin{gathered}
F_{p}=\frac{i \eta}{c_{m} r_{w}} \frac{u}{c_{e}} \\
F_{d}(s, v)=\frac{1}{c_{m}}\left(F_{a}(v)+F_{r}+F_{g}(s)\right)
\end{gathered}
$$

where $F_{a}(v)=\frac{1}{2} c_{w} A_{a} \rho_{a} v^{2}, F_{r}=m g_{0} c_{r}-\frac{i \eta}{r_{w}} d_{e}$, and $F_{g}(s)=m g_{0} \alpha(s)$. The control is bounded by

$$
0 \leq u \leq \bar{u}
$$

where $\bar{u}$ is maximum fueling. The mass flow of fuel (3) is

$$
M^{\prime}=c_{u} u, \quad c_{u}=\frac{n_{c y l}}{2 \pi n_{r}} \frac{i}{r_{w}}
$$

where $c_{u}$ is a constant.

\section{LOOK-AHEAD}

Look-ahead control utilizes RHC to handle the complexity due to changing conditions. Now the RHC problem is formulated, and based on that suboptimality measures are introduced.

\subsection{Receding horizon}

Combining Eqs. (1) and (2), the system model is given by

$$
\frac{d x}{d s}=f(x, u, s), \quad x(0)=x_{0}
$$

where $x \in X, u \in U$ are states and controls. The problem over the entire drive mission $s \in[0, S]$ is defined by

$$
J^{S}\left(x_{0}\right)=\min _{u \in U} \int_{0}^{S} L(x, u) d s
$$

where $L$ is the running cost given by (P2).

Denote by $J^{R}$ the optimal cost for the RHC problem with the horizon length $R$. Let $s_{r}$ be the current position and $P=\min \left\{s_{r}+R, S\right\}$. The problem

$$
J^{R}\left(s_{r}, x\right)=\min _{u \in U}\left\{\phi(x(P))+\int_{s_{r}}^{P} L(x, u) d s\right\}
$$

where $\phi$ is a residual cost, is solved repeatedly on-line by some optimization method. The solution is the RHC controller, denoted by $\mu^{R}$, and the resulting trajectory is the solution for

$$
\frac{d x}{d s}=f\left(x, \mu^{R}, s\right), \quad x(0)=x_{0}
$$

with an associated cost

$$
J_{\mu^{R}}^{S}\left(x_{0}\right)=\int_{0}^{S} L\left(x, \mu^{R}\right) d s
$$

Thus, $J_{\mu^{R}}^{S}$ gives a basis for evaluating a given horizon length $R$ in relation to the full problem given by $J^{S}$ in (9). The other important performance factor in RHC is the residual cost $\phi$ in (10). The choice of $R$ and $\phi$ will be studied in the following.

\subsection{Suboptimality measures}

To begin with, the basis for studying different horizon lengths is introduced. This foundation is valid independent of the choice of the residual cost $\phi$ in (10). 
The difference between the costs (9) and (12) is the central matter, and it is clear that the relation

$$
J^{S}(x) \leq J_{\mu^{R}}^{S}(x)
$$

holds between these costs. To quantify the difference between them, a measure of the suboptimality imposed by $\mathrm{RHC}$ is introduced that is the relative difference between the costs. Consequently, define the degree of suboptimality for a horizon length $R$ by $\kappa_{J}^{R}$, where

$$
\kappa_{J}^{R}=J_{\mu^{R}}^{S}(x) / J^{S}(x)-1
$$

Clearly, $\kappa_{J}^{R}$ is non-negative, is zero for the optimal control law for the original problem (9), and $\kappa_{J}^{R} \rightarrow 0$ when $R \rightarrow S$. The suboptimality is decreasing with horizon length so studying $\kappa_{J}^{R}$ for varying $R$ can be used to judge suitable horizon lengths. Note that the measure is independent of how the optimal solution for (10) is obtained.

The application here is problem (P2) where

$$
J^{S}(x)=M^{S}(x)+\beta T^{S}(x)
$$

To separate the suboptimality in fuel mass $M$ and trip time $T$, define the measures $\kappa_{M}^{R}, \kappa_{T}^{R}$ by

$$
\kappa_{M}^{R}=\frac{M_{\mu^{R}}^{S}(x)}{M^{S}(x)}-1, \quad \kappa_{T}^{R}=\frac{T_{\mu^{R}}^{S}(x)}{T^{S}(x)}-1
$$

analogous to (14). The measures $\kappa_{J}^{R}, \kappa_{M}^{R}, \kappa_{T}^{R}$ are studied quantitatively for (P2) and varying $R$ in Sec. 6 .

\section{FUEL EQUIVALENTS}

For an efficient solution of (P1), fuel equivalents have been introduced in Hellström et al. (2010b,a) based on physical intuition. The support for these is substantiated in Sec. 5 by a mathematical interpretation relating to optimal control theory. The use of fuel equivalents is inspired by the electrical energy equivalents in works by, e.g., Paganelli et al. (2000); Sciarretta et al. (2004).

\subsection{Kinetic energy equivalence - residual cost}

The residual cost, the other important topic in RHC, is now treated. Starting with the fuel equivalent $\gamma(\mathrm{g} / \mathrm{J})$ that is based on that Eq. (4) represents an affine relation between engine torque $T_{e}$ and fueling $u$. The scaled gradient,

$$
\gamma=\frac{n_{c y l}}{2 \pi n_{r} \eta} c_{e}
$$

defines an equivalence between energy and fuel mass.

In Hellström et al. (2010a), the idea is to calculate kinetic energy into an equivalent fuel mass as an approximation of the fact that kinetic energy can be used to save fuel in the future. The residual cost $\phi(x)=-\gamma e$, where $e=\frac{1}{2} m v^{2}$ is kinetic energy at the end of the horizon, was proposed. A slightly modified residual cost is obtained by rewriting the basic model for a small $\Delta s$. Eqs. (1),(5),(7), and (17) yield

$$
\Delta M=\gamma\left[c_{m} \Delta e+\Delta p+\left(F_{a}(v)+F_{r}\right) \Delta s\right]
$$

where $p$ is potential energy and $\Delta p \approx m g_{0} \alpha \Delta s$. From (18) it is seen that a change $\Delta e$ in kinetic energy approximately equals a fuel mass $\gamma c_{m} \Delta e$. Based on this, the mass factor $c_{m}$ should be included to yield

$$
\phi(x)=-\gamma c_{m} e
$$

but $c_{m}$ is typically close to one, especially for large masses and higher gears. The value of $\gamma$ for an example of a typical engine in a heavy diesel truck is $53 \mathrm{~g} / \mathrm{MJ}$ or $4.6 \mathrm{kWh} / \mathrm{L}$.

\subsection{Time equivalence}

The reformulation in (P2) is beneficial since the problem has a lower dimension than (P1) and since, with an RHC approach, it avoids the risk of an infeasible constraint. However, the parameter $\beta$ has to be selected. An approximate value of $\beta$ can be found by assuming that the constant speed $\hat{v}$ is the solution for the trip length $S$. Using $S=\hat{v} T_{0}$ and (18) gives the criterion.

$$
J(\hat{v})=\gamma(p(S)-p(0))+\gamma\left(F_{a}(\hat{v})+F_{r}\right) S+\beta \frac{S}{\hat{v}}
$$

In a stationary point $J^{\prime}(\hat{v})=0$ which yields $\beta=\gamma \hat{v}^{2} F_{a}^{\prime}(\hat{v})$. The air drag force according to Table 1 yield

$$
\beta=2 \gamma P_{a}(\hat{v})=2 \gamma P_{a}\left(S / T_{0}\right)
$$

where $P_{a}(\hat{v})=\hat{v} F_{a}(\hat{v})$ is the air drag power. With $\beta$ according to $(21), J^{\prime \prime}(\hat{v})$ is positive for all physically feasible parameters which shows that it gives a minimum for this stationary case. The compromise between fuel mass and trip time is defined by the ratio

$$
q=\frac{M}{\beta T}=\frac{1}{2}\left(1+\frac{F_{r}}{F_{a}(\hat{v})}\right)
$$

A change in $\beta$ gives another stationary speed $v(\beta)$ and $q$. The relative changes in fuel mass and trip time become

$$
\kappa_{M}^{\beta}=\frac{F_{a}(v(\beta))-F_{a}(\hat{v})}{F_{a}(\hat{v})+F_{r}}, \quad \kappa_{T}^{\beta}=\frac{\hat{v}}{v(\beta)}-1
$$

The slope in the origin of the graph $\kappa_{M}^{\beta}$ versus $\kappa_{T}^{\beta}$ becomes $-q^{-1}$ where $q$ is given by (22) and so, close to the origin

$$
q \kappa_{M}^{\beta}+\kappa_{T}^{\beta}=0
$$

holds. Examples of typical parameters for a heavy diesel truck and $80 \mathrm{~km} / \mathrm{h}$ give $\beta$ as $4.5 \mathrm{~g} / \mathrm{s}$ or $18.5 \mathrm{~L} / \mathrm{h}$ and $q$ as 1.2 . The ratio $q$ thus indicates that an increase in time of $1.2 \%$ gives, approximately, a decrease in fuel mass of $1 \%$.

\section{INTERPRETATION OF FUEL EQUIVALENTS}

The fuel equivalents in Sec. 4 are given a mathematical interpretation by relating them to the Lagrange multipliers used in optimization theory. To accomplish this, first an optimal control problem is formulated and the multipliers for this problem are throughly studied. A related work is Fröberg and Nielsen (2008) where the multiplier for a velocity state is studied.

\subsection{Problem formulation}

Consider (P1) for the basic model in Sec. 2.2 and treat the time constraint as an equality constraint since the minimum is attained in the limit for a realistic drive mission:

$$
\begin{gathered}
\text { minimize } M \\
\text { subject to } T=T_{0}
\end{gathered}
$$

The problem (P3) is specified exactly below by (25)-(28). In the formulation, it is convenient to use position $s$ as independent variable and kinetic energy $e$ as state instead of velocity. The notation in the following is adopted from Bryson and Ho (1975).

The states are time $t$ and kinetic energy $e$ with associated Lagrange multipliers denoted by

$$
x=(t, e)^{T}, \quad \lambda=\left(\lambda_{t}, \lambda_{e}\right)^{T}
$$


The dynamics is

$$
x^{\prime}=f(x, u, s)=\left(\begin{array}{c}
\sqrt{m / 2 e} \\
\frac{c_{u}}{\gamma c_{m}} u-F_{d}
\end{array}\right)
$$

The running cost is $L=M^{\prime}$, so (7) gives the objective

$$
J=\min _{u \in U} \int_{0}^{S} c_{u} u d s
$$

with $U$ given by (6). The trip time constraint is

$$
\psi(x(S))=t(S)-T_{0}
$$

Together with an initial condition

$$
x(0)=\left(0, \frac{1}{2} m v_{0}^{2}\right)
$$

the problem (P3) becomes completely specified.

\subsection{Solution}

The Minimum Principle states that the optimal control minimizes the Hamiltonian $H$,

$$
u^{\star}=\underset{u \in U}{\arg \min } H
$$

where $H=L+\lambda^{T} f$ and

$$
\lambda^{\prime T}=-\frac{\partial H}{\partial x}, \quad \lambda(S)=\nu^{T} \frac{\partial \psi}{\partial x}
$$

where $\nu$ is a constant vector (Bertsekas, 1995, Ch. 3.3).

For the problem (P3), the Hamiltonian is

$$
H=L+\lambda^{T} f=\sigma u+\lambda_{t} \sqrt{m / 2 e}-\lambda_{e} F_{d}
$$

where $\sigma$ is given by

$$
\sigma(s)=\frac{c_{u}}{\gamma c_{m}}\left(\gamma c_{m}+\lambda_{e}(s)\right)
$$

The dynamics of $\lambda(30)$ is

$$
\lambda^{\prime}=\left(\frac{\lambda_{t}}{m}\left(\frac{m}{2 e}\right)^{\frac{3}{2}}+\frac{\lambda_{e}}{c_{m}} \frac{\partial F_{a}}{\partial e}\right), \quad \lambda(S)=\left(\begin{array}{l}
\nu \\
0
\end{array}\right)
$$

where the fact that $\frac{\partial}{\partial e} F_{d}(s, e)=\frac{1}{c_{m}} \frac{\partial}{\partial e} F_{a}(e)$ has been used. Since $H$ is linear in $u$, the solution is at one of the bounds in (6) if $\sigma(s) \neq 0$. If $\sigma(s)=0$, the solution is singular and can not be determined from the Minimum Principle. To summarize, the possible controls are

$$
u^{\star}= \begin{cases}0 & \sigma(s)>0 \\ \hat{u} & \sigma(s)=0 \\ \bar{u} & \sigma(s)<0\end{cases}
$$

where $\hat{u}$ is the yet unknown control on a singular arc.

To find $\hat{u}$, the singular arc is studied. Eq. (32) immediately gives that if $\sigma(s)=0$, then

$$
\lambda_{e}(s)=-\gamma c_{m}
$$

If $\sigma(s)=0$ on a finite interval, it must hold that $\sigma^{\prime}(s)=0$. From (32) and (33) together with (35) it then follows that

$$
\sigma^{\prime}=\frac{\lambda_{t}}{m}\left(\frac{m}{2 e}\right)^{\frac{3}{2}}-\gamma \frac{\partial F_{a}}{\partial e}=0
$$

which shows that, since $\lambda_{t}$ and $\frac{\partial F_{a}}{\partial e}$ are constant, the kinetic energy is constant on a singular arc (this further implies that $\left.\sigma^{\prime \prime}(s)=0\right)$. The control $\hat{u}$ is then given from (25)

$$
\hat{u}=\frac{\gamma c_{m}}{c_{u}} F_{d}(s, \hat{v})
$$

where $\hat{v}$ is the constant speed. Singular arcs are possible on road segments with small slopes such that constant speed is feasible since there must be a feasible $\hat{u}, 0 \leq \hat{u} \leq \bar{u}$.

Solving (36) for $\lambda_{t}$ and inserting the air drag force $F_{a}$ in (5b) shows that, on a singular arc,

$$
\lambda_{t}=2 \gamma P_{a}(\hat{v})
$$

holds where $P_{a}(\hat{v})=\hat{v} F_{a}(\hat{v})$ is the air drag power. Since $\nu=\lambda_{t}$ is constant, the choice of $\nu$ such that the trip time constraint is satisfied also determines the constant speed $\hat{v}$ on singular arcs.

A complete solution for (P3) is given by solving the twopoint boundary value problem given by (25), (28), and (33) where $\nu$ is determined by the trip time constraint (27).

\subsection{Interpretation}

The relationships (Bryson and Ho, 1975; Bertsekas, 1995)

or equivalently

$$
\lambda^{T}=\frac{\partial J}{\partial x}, \quad H=-\frac{\partial J}{\partial s}
$$

$$
d J=\lambda^{T} d x-H d s
$$

form a general connection between the optimal cost function $J$ and the Lagrange multipliers $\lambda$ and the Hamiltonian $H$. The aim here is to investigate physically meaningful interpretations of these quantities.

The control $u$ may be discontinuous if selected according to (34). Moreover, the road slope $\alpha(s)$ is typically known in discrete points $\alpha_{k}$ where

$$
\alpha(s)=\alpha_{k}, \quad s \in[k \Delta s,(k+1) \Delta s)
$$

Jumps in $\alpha(s)$ and $u(s)$ yield jumps in the system dynamics $f$, and the point at which $f$ changes can be seen as an interior boundary condition. Since, in this case, the condition becomes a function only of position, it leads to that $\lambda$ is continuous whereas $H$ may be discontinuous (see Bryson and Ho, 1975, Ch. 3.5).

Kinetic energy The dynamics of $\lambda_{e}$ is written as

$$
\lambda_{e}^{\prime}(s)=\theta \lambda_{e}(s)+\gamma c_{m} \theta\left(\frac{\hat{v}}{v(s)}\right)^{3}, \quad \theta=\frac{c_{w} A_{a} \rho_{a}}{c_{m} m}
$$

by inserting (5b) and (38) into (33). It turns out that the adjoint dynamics is driven by the deviation of the optimal velocity $v(s)$ from the constant level $\hat{v}$ and it is expected that $\lambda_{e}(s)$ varies around $-\gamma c_{m}$ if $v(s)$ varies around $\hat{v}$. On a singular arc, $v(s)=\hat{v}$ which implies that $\lambda_{e}^{\prime}$ is zero.

A variation $\delta e$ on an optimal trajectory gives a changed cost $\delta J=\lambda_{e} \delta e$ according to (39), i.e., a change in kinetic energy leads to a proportional change in the equivalent fuel consumption with the constant of proportionality being $\lambda_{e}$. In particular, on a singular arc, $\delta J=-\gamma c_{m} \delta e$ and

$$
\left.\frac{\partial J}{\partial e}\right|_{e=\hat{e}}=-\gamma c_{m}, \quad \hat{e}=\frac{1}{2} m \hat{v}^{2}
$$

The change in the Hamiltonian (31) due to a change in the road slope at $s=s_{1}$ becomes, since $x$ and $\lambda$ are continuous,

$$
H\left(s_{1}+\right)-H\left(s_{1}-\right)=-\frac{1}{c_{m}} \lambda_{e}\left(s_{1}\right) m g_{0}\left(\alpha_{k}-\alpha_{k-1}\right)
$$

The Hamiltonian is therefore stepwise constant

$$
H(s)=H_{k}, \quad s \in[k \Delta s,(k+1) \Delta s)
$$


and may be written as

$$
H_{k}=H_{0}-\frac{1}{c_{m}} \sum_{j=1}^{k} \lambda_{e}(j \Delta s) m g_{0}\left(\alpha_{j}-\alpha_{j-1}\right)
$$

for $k=1,2, \ldots, S / \Delta s-1$. According to (40), the change in the optimal cost due to a $\Delta s$ with $\Delta x=0$ is $-H$. Potential energy is approximately $m g_{0} \alpha(s) \Delta s$ and consequently, $\lambda_{e}(s)$ determines the proportional change in the cost due to the change in potential energy during $[s, s+\Delta s)$.

Time When solving (P3), the value of $\nu=\lambda_{t}=2 \gamma P_{a}(\hat{v})$ must be found such that the trip time constraint (27) is satisfied. Using the time equivalent $\beta$ in (21) is the same as choosing a value $\beta$, removing the trip time constraint (27) by letting $\psi=0$, and modify the objective (26) as

$$
J=\min _{u \in U} \int_{0}^{S} c_{u} u+\frac{\beta}{v} d s
$$

that is equal to the formulation (P2). This formulation may lead to a trip time $T \neq T_{0}$. With the original formulation, a variation $\delta t$ on the optimal trajectory gives a changed $\operatorname{cost} \delta J=\nu \delta t$ according to (39) and $\nu$ is thus a measure of the increase in the equivalent fuel consumption if the remaining time decreases.

Summary In conclusion, $\lambda_{t}$ determines the constant speed $\hat{v}$ on singular arcs, according to (38), whereas $\lambda_{e}$ is the decisive variable for the dynamical behavior, i.e., when the velocity deviates from $\hat{v}$, according to (32),(34),(42). The standard interpretation, given by (39), of the values of the adjoint variables is that they are the gradient of the cost function with respect to the states. In addition, it turns out in (46) that, in the position direction, the cost function varies proportional to the varying potential energy with the constant of proportionality equal to $\lambda_{e}$.

\subsection{Residual cost}

The residual cost (19) is now interpreted by aid of problem (P3). Consider an RHC approach for solving (P3) by using the objective (47) and $\psi=0$. The real residual cost for the objective (47) at position $s$, with $x=(t, e)$, is

$$
J(s, x)=\min _{u \in U} \int_{s}^{S} c_{u} u+\beta t^{\prime} d s
$$

Since $\psi=0,(30)$ yields that $\lambda_{t}=0$, so it follows from (39) that $J(s, x)$ is a function of $s$ and $e$. Eq. (39) gives that

$$
\frac{\partial J}{\partial e}=\lambda_{e}
$$

According to (35), $\lambda_{e}=-\gamma c_{m}$ on a singular arc and now, approximate $\lambda_{e}$ with this constant value on constrained arcs as well, i.e., $\lambda_{e} \approx-\gamma c_{m}$. Integration of (39) with respect to $e$ then gives

$$
J(s, x) \approx-\gamma c_{m} e+C(s)
$$

The integration constant $C(s)$ does not affect the optimal solution and can be omitted when choosing a residual cost $\phi$. Thus, this connection to optimal control theory supports the choice of the residual cost $\phi(x)=-\gamma c_{m} e$ in (19). Further, the choice is justified in Hellström et al. (2010a) where it is shown that, without approximations, the real residual cost (48) is dominated by this term.
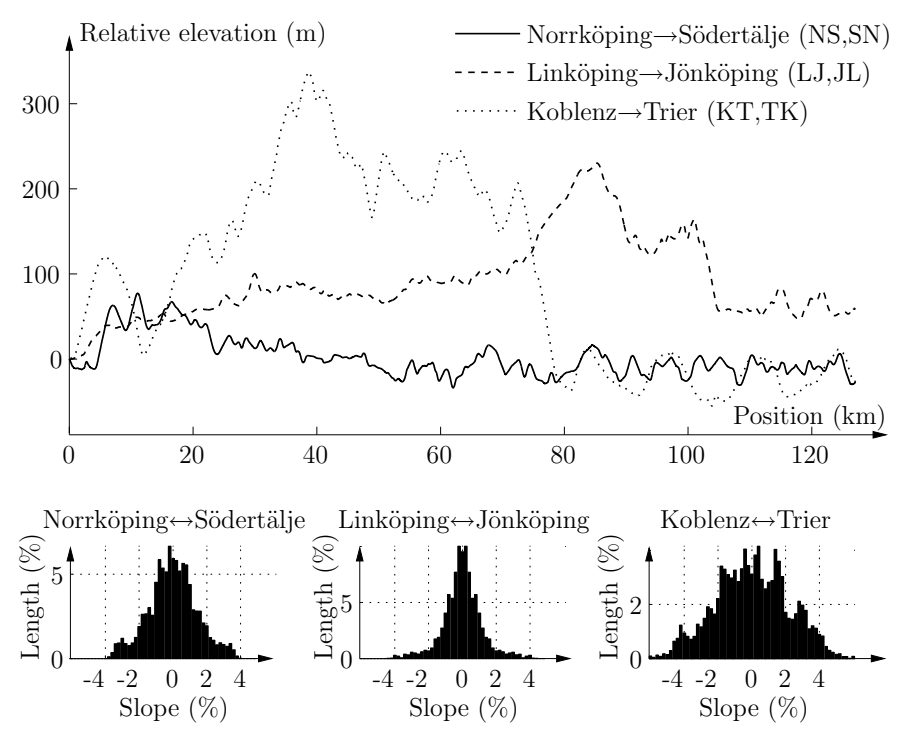

Fig. 2. Elevation profiles (in one direction) and distributions of road slope values (in both directions) for two Swedish routes and one German route.

\section{QUANTITATIVE STUDY}

The choice of horizon length is quantitatively studied by evaluating the suboptimality measures in Sec. 3.2. The full model in Sec. 2.1 and three different routes, with the characteristics and the abbreviations in Fig. 2, are used. The maximum allowed speed is $89 \mathrm{~km} / \mathrm{h}$ and $\beta$ in (P2) is chosen for a cruising speed of $\hat{v}=84 \mathrm{~km} / \mathrm{h}$. The vehicle parameters are from the experimental setup in Hellström et al. (2009) and represent a truck with a gross weight of $40 \mathrm{t}$ with a relatively small engine of $310 \mathrm{hp}$. The computation of the optimal cost functions (9) and (10) is done by value iteration (see, e.g., Bertsekas, 1995) by utilizing the algorithm development in Hellström et al. (2010a).

In Fig. 3, the measure $\kappa_{J}^{R}$ is shown for different horizon lengths $R$. It is seen that $\kappa_{J}^{R}$ depends on the route but the rate of decrease, i.e., the relative benefit of increasing $R$, for around $1-2 \mathrm{~km}$ is similar. To study the effect on the fueltime trade-off, Fig. 4 shows $\kappa_{M}^{R}$ versus $\kappa_{T}^{R}$ for increasing $R$. It is observed that optimality is approached approximately along a line with negative slope. To explain this behavior, note the following relationship between the suboptimality measures obtained by combining (13)-(16):

$$
0 \leq \kappa_{J}^{R}(1+q)=q \kappa_{M}^{R}+\kappa_{T}^{R}, \quad q=\frac{M^{S}}{\beta T^{S}}
$$

Now, since $\kappa_{J}^{R}$ tends to zero faster than the other terms, the solution approaches the line $q \kappa_{M}^{R}+\kappa_{T}^{R}=0$. By computing $J^{S}$ in (15) for varying $\beta$, it shows that the ratio $q$ is around 1 which explains the observed behavior. The computations also show that Eq. (24) gives a good approximation when $\kappa_{M}^{\beta}, \kappa_{T}^{\beta}$ are a few percent. With a desired suboptimality $d$ in $M$, it is reasonable, based on (24), to aim for

$$
0 \leq q \kappa_{M}^{R}+\kappa_{T}^{R} \leq d
$$

since this is close to an optimal solution with a different $\beta$. The optimal trade-off line (24) and the desired area (52) are shown in Fig. 4 for $d=0.5 \%$ and $q$ given by (22). These are used to determine horizon lengths with the appropriate compromise between fuel consumption and trip time. 


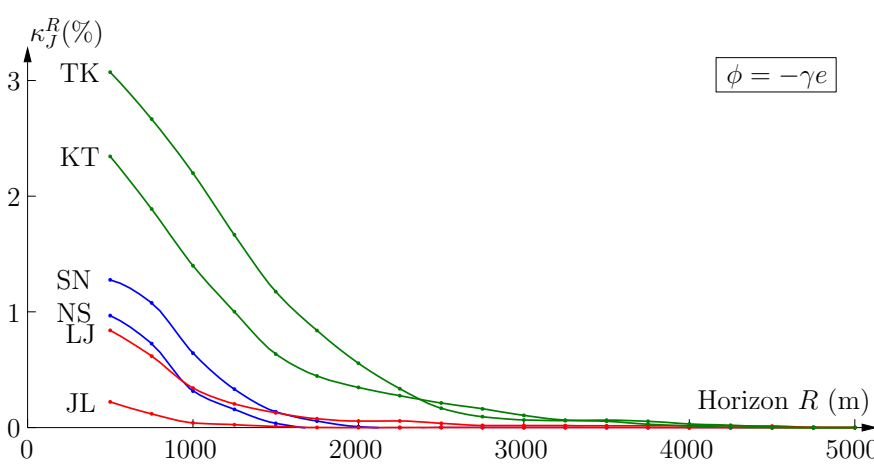

Fig. 3. Suboptimality for varying horizon length.

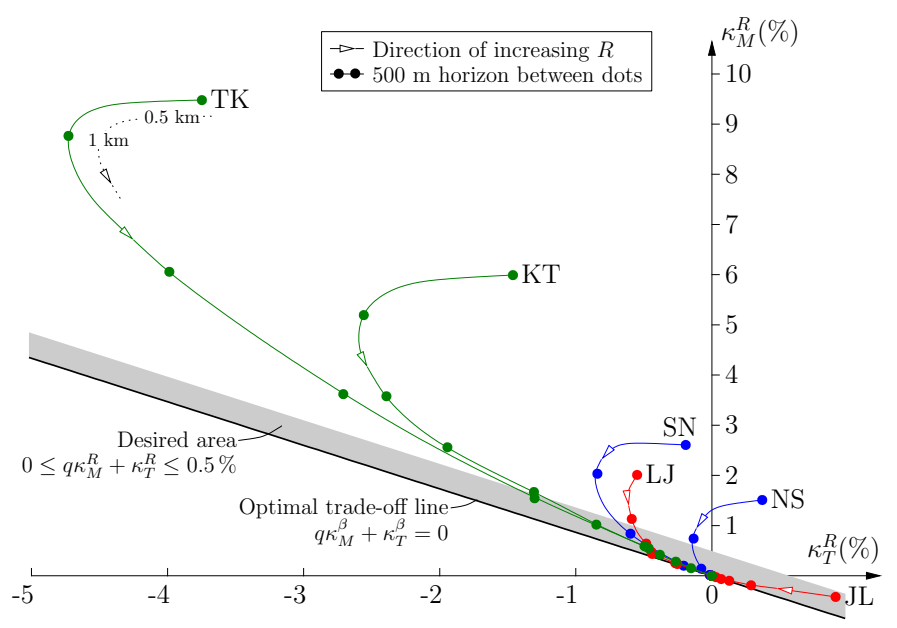

Fig. 4. The suboptimality in fuel and time.

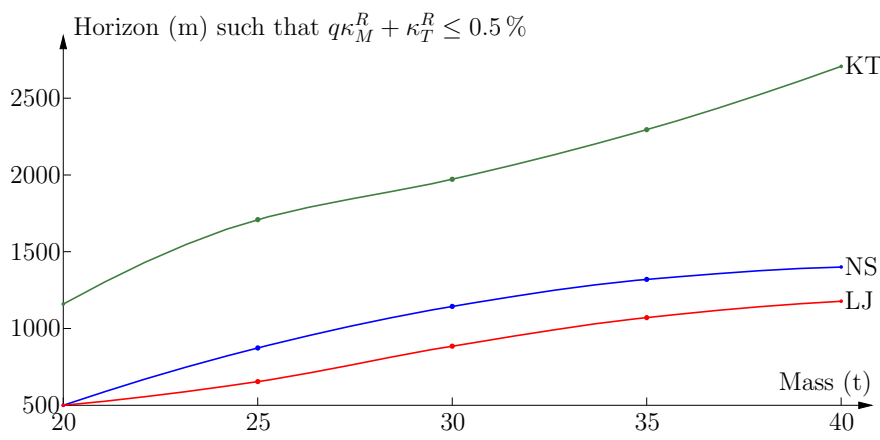

Fig. 5. Required horizon to obtain (52) with $d=0.5 \%$.

The vehicle mass is now varied by repeating the computations for lower masses down to $20 \mathrm{t}$. The principle behavior in Figs. 3-4 remains the same but the necessary horizon length to reach a certain degree of suboptimality decreases with decreasing mass. This effect is shown in Fig. 5 where the necessary horizon length to reach the desired area (52) for $q$ given by (22), in both directions of the respective route, is drawn as a function of vehicle mass.

\section{CONCLUSIONS}

Approximating the residual cost and choosing the horizon length are the two main issues in RHC, and these are addressed here for the look-ahead problem. The support for the residual cost used is strengthened by a mathematical interpretation, in terms of Lagrange multipliers, that confirms the physical intuition. The choice of horizon length is a compromise between complexity and suboptimality, and this compromise is quantified by introducing measures for suboptimality. These are combined into an optimal trade-off line that enables choosing horizon lengths with the appropriate compromise between fuel consumption and trip time. Altogether, the framework for quantitative analysis provide valuable insights into design and tuning for different road characteristics and vehicle mass.

\section{REFERENCES}

Bertsekas, D. (1995). Dynamic Programming and Optimal Control, volume I. Athena Scientific, Belmont, Mass.

Bryson, A.E. and Ho, Y. (1975). Applied Optimal Control. Taylor and Francis.

Fröberg, A. and Nielsen, L. (2008). Optimal control utilizing analytical solutions for heavy truck cruise control. Technical Report LiTH-ISY-R-2842, Dept. of Electrical Engineering, Linköping University, Sweden.

Guzzella, L. and Sciarretta, A. (2005). Vehicle Propulsion Systems - Introduction to Modeling and Optimization. Springer-Verlag, Berlin.

Hellström, E., Ivarsson, M., Åslund, J., and Nielsen, L. (2007). Look-ahead control for heavy trucks to minimize trip time and fuel consumption. In 5th IFAC Advances in Automotive Control. Monterey, CA, USA.

Hellström, E., Ivarsson, M., Åslund, J., and Nielsen, L. (2009). Look-ahead control for heavy trucks to minimize trip time and fuel consumption. Control Engineering Practice, 17(2), 245-254.

Hellström, E., Åslund, J., and Nielsen, L. (2010a). Design of an efficient algorithm for fuel-optimal lookahead control. Control Engineering Practice, in press, doi:10.1016/j.conengprac.2009.12.008.

Hellström, E., Åslund, J., and Nielsen, L. (2010b). Management of kinetic and electric energy in heavy trucks. In SAE World Congress, number 2010-01-1314 in SAE Technical Paper Series. Detroit, MI, USA.

Huang, W., Bevly, D., Schnick, S., and Li, X. (2008). Using $3 \mathrm{D}$ road geometry to optimize heavy truck fuel efficiency. In 11th International IEEE Conference on Intelligent Transportation Systems, 334-339.

Kiencke, U. and Nielsen, L. (2005). Automotive Control Systems, For Engine, Driveline, and Vehicle. SpringerVerlag, Berlin, 2nd edition.

Mayne, D.Q., Rawlings, J.B., Rao, C.V., and Scokaert, P.O.M. (2000). Constrained model predictive control: Stability and optimality. Automatica, 36(6), 789-814.

Paganelli, G., Guerra, T., Delprat, S., Santin, J.J., Delhom, M., and Combes, E. (2000). Simulation and assessment of power control strategies for a parallel hybrid car. Proc. of the Institution of Mechanical Engineers - Part D Journal of Automobile Engineering, 214(7), 705-717.

Passenberg, B., Kock, P., and Stursberg, O. (2009). Combined time and fuel optimal driving of trucks based on a hybrid model. In European Control Conference. Budapest, Hungary.

Sciarretta, A., Back, M., and Guzzella, L. (2004). Optimal control of parallel hybrid electric vehicles. IEEE Transactions on Control Systems Technology, 12(3), 352-363.

Terwen, S., Back, M., and Krebs, V. (2004). Predictive powertrain control for heavy duty trucks. In 4 th IFAC Advances in Automotive Control. Salerno, Italy. 